\title{
Improved physical properties of the quadruple sub-system with the eclipsing binary QZ Carinae*
}

P. Mayer ${ }^{1 \dagger}$, P. Harmanec ${ }^{1}$, P. Zasche ${ }^{1}$, R. Catalan-Hurtado ${ }^{2}$, B.N. Barlow ${ }^{2}$, Y. Frémat ${ }^{3}$, M. Wolf ${ }^{1}$, H. Drechsel ${ }^{4}$, R. Chini ${ }^{5,6}$, A. Nasseri ${ }^{5}$, G.W. Christie ${ }^{7}$, W.S.G. Walker ${ }^{8}$, A.A. Henden ${ }^{9}$, T. Bohlsen ${ }^{10}$ and H. Božić ${ }^{11}$

1 Astronomical Institute of the Charles University, Faculty of Mathematics and Physics,

V Holešovičkách 2, CZ-180 00 Praha 8, Czech Republic

${ }^{2}$ Department of Physics, High Point University, One University Way, High Point, NC 27268, USA

3 Royal Observatory of Belgium, Ringlaan 3, B-1180 Brussel, Belgium

4 Dr. Karl Remeis-Observatory \& ECAP, Astronomical Institute, Friedrich-Alexander-University Erlangen-Nuremberg, Sternwartstr. 7, 96049 Bamberg, Germany

5 Astronomisches Institut, Ruhr-Universität Bochum, Universitätsstr. 150, 44801 Bochum, Germany

${ }^{6}$ Instituto de Astronomía, Universidad Católica del Norte, Avenida Angamos 0610, Antofagasta, Chile

7 Auckland Observatory, PO Box 24180, Royal Oak, Auckland, New Zealand

8 Variable Stars South, P O Box 173, Awanui, New Zealand, 0451

9 AAVSO, 106 Hawking Pond Road, Center Harbor, NH03226, USA

10 Mirranook Observatory, Boorolong Rd Armidale, NSW, 2350, Australia

11 Hvar Observatory, Faculty of Geodesy, Zagreb University, Kačićeva 26, 10000 Zagreb, Croatia

Received: October 1, 2019; Accepted: December 2, 2019

\begin{abstract}
Using a collection of 79 spectra of the quadruple system QZ Car, we were able - for the first time in the optical region - to measure radial velocities of both components of the eclipsing subsystem Ac, and in combination with the photometric solution to obtain masses and radii of both bodies. We confirm that the Ac binary is a semi-detached system with a brighter primary Ac1 and a more massive secondary Ac2. Variations due to the light-time effect and secular
\end{abstract}

* Based on spectra from observations made with ESO telescopes at La Silla and Paranal Observatories under programmes 076.C-0431(A), 081.C-2003(A), 083.D-0589(A), 089.D0975(A), 095.A-9032(A); the program TYCHO P2; on the BESO spectra and spectra from CTIO, and on Hipparcos, ASAS3 and our $V$ photometry.

$\dagger$ Pavel Mayer passed away on the day of his $86^{\text {th }}$ birthday, Nov. 7, 2018 
changes in the systemic velocities of both binaries, Aa and Ac, allowed us to estimate the period of mutual orbit of binaries Aa and Ac to be about $11700 \mathrm{~d}$. Preliminary improved basic properties of the system and its components are provided.

Key words: Stars: binaries: eclipsing - Stars: early-type - Stars: fundamental parameters - Stars: individual: QZ Car

\section{Introduction}

The massive multiple system known as HD 93206 (HIP 52526), a member of the open cluster Collinder 228, is known to consist of at least eight stars, four of them belonging to two binary systems in a mutual orbit, known as QZ Car. Components Aa1 and Aa2 form a spectroscopic binary $(P=20 \mathrm{~d} 7, e=0.35)$ while components Ac1 and Ac2 form an eclipsing binary $(P=5$ d.9987, $e=0)$. There is also a close component $\mathrm{Ab}^{1}$ and more distant faint components $\mathrm{B}, \mathrm{C}$, and D - see Fig. 1.

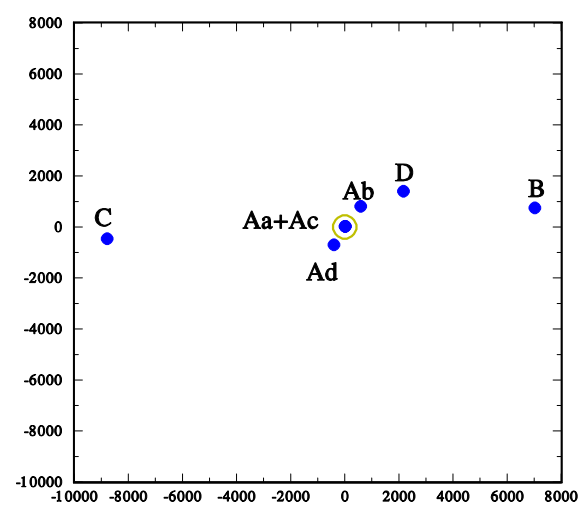

Figure 1. A sketch of the multiple system HD 93206 drawn in mas scale on both axes.

QZ Car was identified as an eclipsing binary by Walker \& Marino (1972). Morrison \& Conti (1979) reported the presence of two spectroscopic binaries in the spectra of the star, the secondary Aa2 being too faint to be detected. Mayer et al. (2001) improved the ephemerides of both orbits and derived new orbital elements for them. Considering the light-time effect they suggested the period of mutual orbit of the Aa and Ac binaries to be 40 to 50 yrs. Stickland \& Lloyd (2001) obtained radial velocities (RVs) of components Aa1, Ac1, and Ac2 from 9 SPW IUE spectra and derived new orbital elements of both orbits. For the Ac1-Ac2 subsystem, they obtained a mass ratio $M_{1} / M_{2}=1.07$. So far the

\footnotetext{
${ }^{1}$ Another close component named Ad was reported during this conference - see Reggiani, Rainot and Sana.
} 

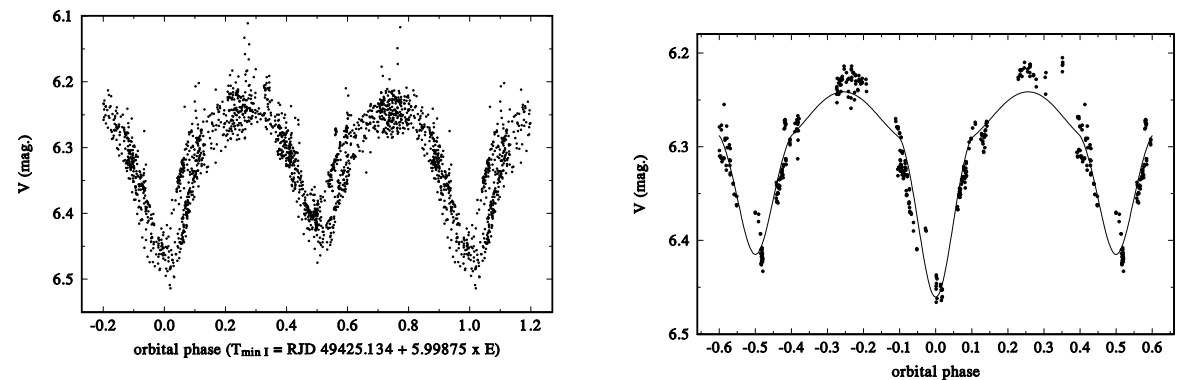

Figure 2. Left: Light curve of eclipsing system Ac based on all homogenised yellow-band observations. Right: Standard $V$ observations fitted with PHOEBE.

most detailed study was published by Walker et al. (2017) who list references to numerous previous studies.
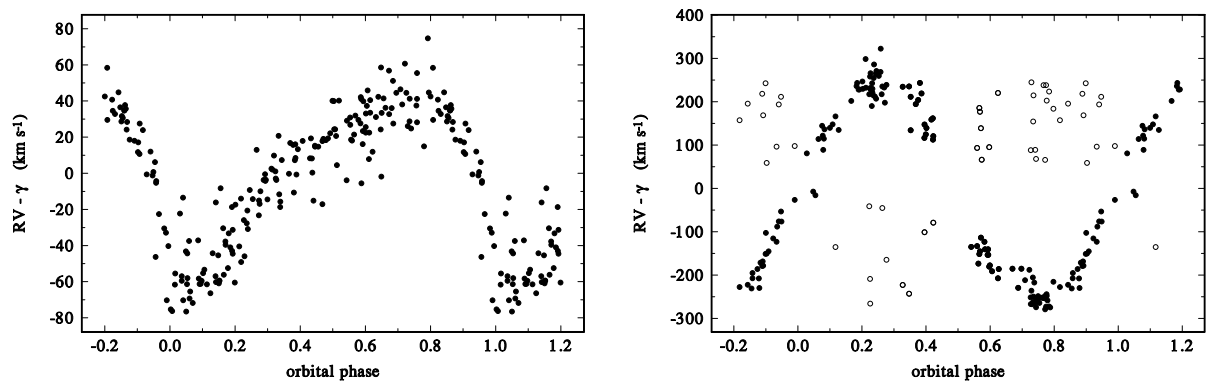

Figure 3. RV curves of component Aa1, and components Ac1 and Ac2.

\section{Our analyses and results}

We reduced and analysed 79 digital spectra from the FEROS, BESO, and $\mathrm{CH}-$ IRON echelle spectrographs, and measured RVs in them. We also collected and homogenised all photometric observations available to us, including the discovery observations by Walker \& Marino (1972). Using all yellow photometry, we derived the mean period of the Ac system as 5.99875. The light curve in the left panel of Fig. 2 shows the changes in the local times of minima due to the light-time effect. We derived new orbital solutions for both subsystems with the program FOTEL (Hadrava, 2004a) in such a way that we allowed calculation of individual $\gamma$ velocities over intervals not longer than about $100 \mathrm{~d}$. The solutions 
Table 1. New orbital solution for all RVs of component Aa1 with locally derived systemic $(\gamma)$ velocities, and the same for components Ac1 and Ac2. All epochs are in HJD-2400000.0. The rms values are for a single observation.

\begin{tabular}{lrr}
\hline \hline Element & \multicolumn{1}{c}{ Aa1 } & Ac1 \& Ac2 \\
\hline$P(\mathrm{~d})$ & $20.73514(33)$ & $5.998728(22)$ \\
$T_{\text {periastr. }}$ & $42529.54(36)$ & - \\
$T_{\text {upper.conj. }}$ & 42528.50 & $49425.031(24)$ \\
$T_{\text {max.RV }}$ & 42524.26 & - \\
$e$ & $0.351(34)$ & 0.0 fixed \\
$\omega\left(^{\circ}\right)$ & $128.5(7.2)$ & - \\
$K_{1}\left(\mathrm{~km} \mathrm{~s}^{-1}\right)$ & $52.3(1.9)$ & $256.8(4.9)$ \\
$K_{2}\left(\mathrm{~km} \mathrm{~s}^{-1}\right)$ & - & 193.5 \\
$M_{2} / M_{1}=K_{1} / K_{2}$ & - & $1.327(90)$ \\
No. of RVs & 203 & 176 \\
rms $\left(\mathrm{km} \mathrm{s}^{-1}\right)$ & 11.98 & 43.62 \\
\hline \hline
\end{tabular}
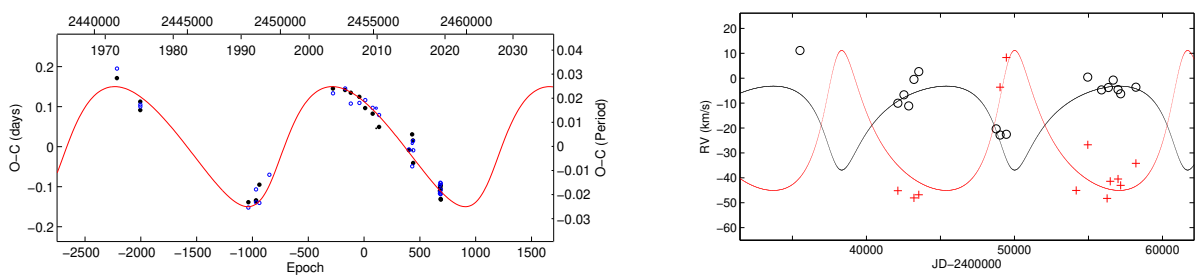

Figure 4. Left: The $O-C$ variations due to the light-time effect plotted vs. phase with the $11700 \mathrm{~d}$ period. Right: Systemic velocities of component Aa1 (circles) and component Ac1 (crosses) plotted with the same period.

are in Table 1 and the corresponding phase curves in Fig. 3. It is encouraging that the RV solution for the binary Ac led to the same mean period as photometry within the error limits.

We derived a mean light-curve solution with PHOEBE 1 (Prša \& Zwitter, 2005) and used it as a template to derive local epochs of minima from subsets of time sorted data. The $O-C$ solution is in the left panel of Fig. 4 while the right panel shows the model fit to the systemic velocities.

Keeping all elements of all three orbits fixed, we disentangled the line profiles of components Aa1, Ac1, and Ac2 with the program KOREL (Hadrava, 2004b) and derived their radiative parameters from the comparison with interpolated synthetic spectra using PYTERPOL. ${ }^{2}$ Fixing the resulting $T_{\text {eff }}$ in PHOEBE 1 , we

${ }^{2}$ Kindly provided by J. Nemravová. 
Table 2. Masses, radii, effective temperatures, relative luminosities, and projected rotational velocities of components Aa1, Ac1, and Ac2 based on our preliminary analysis. The orbital inclination and semi-major axis of the eclipsing system Ac are $i=73^{\circ} .18 \pm 00^{\circ} .16$, and $a=55.77 \pm 0.87 \mathcal{R}_{\odot}^{\mathrm{N}}$, respectively.

\begin{tabular}{lrrr}
\hline \hline Quantity & Aa1 & Ac1 & Ac2 \\
\hline$M\left(\mathcal{M}_{\odot}^{\mathrm{N}}\right)$ & - & 27.8 & 36.9 \\
$R\left(\mathcal{R}_{\odot}^{\mathrm{N}}\right)$ & - & 19.8 & 15.2 \\
$T_{\text {eff }}(\mathrm{K})$ & 32700 & 32700 & 38440 \\
$L$ & 0.42 & 0.32 & 0.24 \\
$v \sin i\left(\mathrm{~km} \mathrm{~s}^{-1}\right)$ & 85 & 134 & 342 \\
\hline \hline
\end{tabular}

arrived at the improved properties of the system listed in Table 2 .

Acknowledgements. The research of $\mathrm{PH}$ and MW was supported by the grant GA19-01995S of the Czech Science Foundation.

\section{References}

Hadrava, P., FOTEL 4 - User's guide. 2004a, Publ. Astron. Inst. Acad. Sci. Czech Rep., 92, 1

Hadrava, P., KOREL - User's guide. 2004b, Publ. Astron. Inst. Acad. Sci. Czech Rep., 92, 15

Mayer, P., Lorenz, R., Drechsel, H., \& Abseim, A., The early-type multiple system QZ Carinae. 2001, Astron. Astrophys., 366, 558, DOI: 10.1051/0004-6361:20000228

Morrison, N. D. \& Conti, P. S., The O-type spectroscopic binary system HD 93206. 1979, in IAU Symposium, Vol. 83, Mass Loss and Evolution of O-Type Stars, ed. P. S. Conti \& C. W. H. De Loore, 277-280

Prša, A. \& Zwitter, T., A Computational Guide to Physics of Eclipsing Binaries. I. Demonstrations and Perspectives. 2005, Astrophys. J., 628, 426, DOI: $10.1086 / 430591$

Stickland, D. J. \& Lloyd, C., Spectroscopic binary orbits from ultraviolet radial velocities. Paper 31: Stars with few IUE observations. 2001, The Observatory, 121, 1

Walker, W. S. G., Blackford, M., Butland, R., \& Budding, E., Absolute parameters of young stars: QZ Carinae. 2017, Mon. Not. R. Astron. Soc., 470, 2007, DOI: $10.1093 / \mathrm{mnras} / \mathrm{stx} 1153$

Walker, W. S. G. \& Marino, B. F., HD 93206 (CSV 6797) an Eclipsing System Presenting Observational Problems. 1972, Information Bulletin on Variable Stars, 681 